\title{
Delicacy Management on Kiloton Dry Wet Spinning Bath Liquid
}

\author{
Fang Liu*, Dong Liu, Pengzong Guo, Guo Li, Rui Yang \\ Zhongfushenying Carbon Fiber Co., Ltd., Lianyungang, China \\ Email address: \\ vanbowling@126.com (Fang Liu) \\ ${ }^{*}$ Corresponding author
}

\section{To cite this article:}

Fang Liu, Dong Liu, Pengzong Guo, Guo Li, Rui Yang. Delicacy Management on Kiloton Dry Wet Spinning Bath Liquid. International Journal of Materials Science and Applications. Vol. 10, No. 1, 2021, pp. 12-17. doi: 10.11648/j.ijmsa.20211001.13

Received: January 12, 2021; Accepted: February 2, 2021; Published: February 26, 2021

\begin{abstract}
During the dry-jet wet spinning process of polyacrylonitrile carbon fiber precursor, the fluctuation of the coagulation bath liquid level affects the stability of the nozzle directly. The motion trajectory and the motion intensity in all directions of the fluid during the movement of the fiber in the coagulation bath fluid field were studied. A three-dimensional model of the cross flow and jet collision motion trajectory was established, and the impact of fluids with different strengths on the surface of the coagulation bath was analyzed. Solidification of the liquid surface in the fitting strength of the peak effect of the return wave overflow trough coupled superimposed to determine the coagulation bath surface to eliminate interfering fluctuations affecting factors. Based on the above analysis, a smart device for real-time monitoring of the coagulation bath air layer has been developed by using the damping equipment in the coagulation bath, where the impact of various fluid waves on the liquid surface can be eliminated on fixed point or position. According to the understanding of kiloton dry-jet wet spinning precursor fiber production line, in-depth exploration has been made to control the high dynamic coagulation bath level effectively from the perspective of technology and delicacy management.
\end{abstract}

Keywords: Dry-jet Wet Spinning, Coagulating Bath Level, Smart Device, Delicacy Management

\section{Introduction}

Carbon fiber is a material with a high degree of stiffness, tensile strength and temperature tolerance as well as low weight and low thermal expansion, all of which make carbon fiber materials popular in the fields of aerospace, civil engineering, military and motor sports [1-2]. Carbon fiber production technology is mainly divided into wet-jet wet spinning and dry-jet wet spinning. Dry-jet spray wet spinning is an advanced spinning technique for preparing high-performance filament. It was first developed by Dongli, Japan in the 1980s. Compared with traditional wet-jet wet spinning, dry-jet wet spinning has the advantages of large drawing ratio of the spinneret, fast spinning speed, and a high orientation degree in fiber [3-4]. At present, the technology has formed a large industrial production worldwide and the industrialization development of kiloton production line has also been realized in some Chinese enterprises. However, there is still a big gap between domestic enterprises and foreign well-known enterprises such as Dongli, Dongbang in terms of technology maturity or in the fine management of carbon fiber process [5-6]. Therefore, it is very important to discuss refinement and quality on the basis of engineering.

The stability of the spray head is directly affected by the fluctuation of the solidification bath liquid surface during the production of dry-jet wet spinning polyacrylonitrile carbon fiber raw wire, and the spray head can easily absorb water and cause serious slurry drop problems [7]. The drafting performance of the wire bundle changes in the air layer and the coagulation bath, and the solidification molding process of the single wire changes accordingly. Thereby the uniformity of the single wire and the quality of the precursor fiber will be affected [8-9].

In dry-jet wet spinning kiloton precursor fiber production line, the amount of single solidification bath in whole line is over 80 . The solidification bath liquid level fluctuation causes water absorption and the production of broken end of the fiber, which will interfere with the operation of other spinning 
positions. So, the stability of the whole line production cannot be controlled and the overall quality of precursor fiber will be reduced. For this reason, how to control the liquid surface of high dynamic solidification bath and keep the liquid level stationary is the key point for the production control of kiloton dry-jet wet spinning carbon fiber raw wire.

In this paper, the flow patterns of the fluid in the solidification bath are analyzed based on the structure of the tank and the trajectory of the fiber. The collision trajectory between the cross flow and the jet is simulated. Based on the experience in the kiloton dry-jet wet spinning raw silk production line of our enterprise, in-depth exploration has been made to control the high dynamic coagulation bath level effectively from the perspective of technology and delicacy management.

\section{Discussion on Influence of Liquid Level Fluctuation in Solidification Bath}

\subsection{Effect of Fluid Movement on Liquid Level Stability in Solidification Bath}

The movement mode of fluid in the solidification bath is influenced by the tow movement. The motion of the tow drives the fluid to move along the direction of the tow, so the speed of the tow movement has a great influence on the motion rate of the fluid in the tank, but with the acceleration of the velocity, the influence of the interference fluid on the fluctuation of the liquid level increases. A detailed trend of fluid movement in the solidification bath is shown in Figure 1 [10].

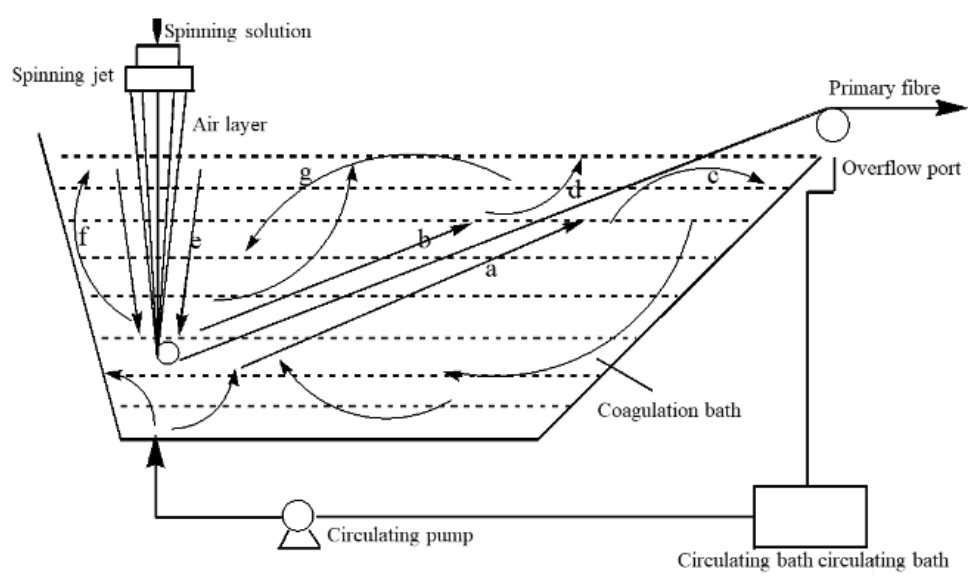

Figure 1. Model diagram of fluid motion in solidification bath.

It can be seen from Figure 1 that with the motion of the tow under traction, the direction of fluid motion in each region also changes. As the a, b, c, d, e fluid moves in the direction of the tow, the flow rate is similar to that of the tow. This part of the fluid is more regular to the direction, the impact on the liquid level is less. But the fluid $\mathrm{g}$, f movement is not regular, it is mainly rising fluid and cyclotron fluid, the movement of these two fluids has a great influence on the liquid level, which directly affects the fluctuation of the area under the nozzle, and has a serious impact on production.

\subsection{Influence of Fluid Collision on Stability of Liquid Level}

From the analysis in Figure 1, it can be seen that the regularity of fluid motion in the groove is quite different, and the collision between the fluids is inevitable in the groove. After the fluid collides with each other under the rate difference, it will appear in many forms, which will inevitably have the form of influence on the liquid level.

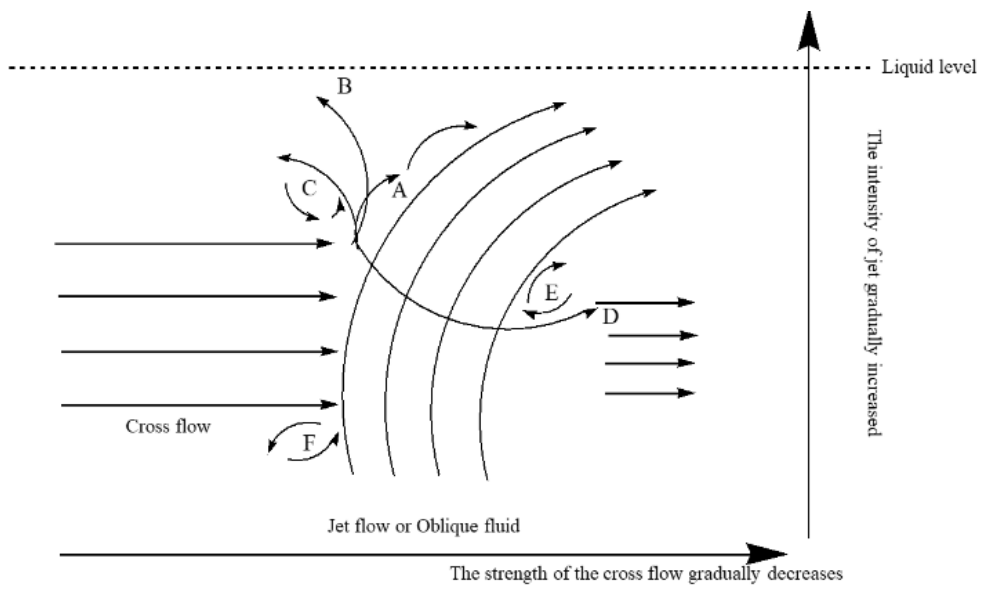

Figure 2. Collision Diagram of Crossflow and Oblique Flow. 
Figure 2 shows the collision between crossflow and oblique fluid in the groove. As can be seen from Figure 2, after a crossflow fluid collides with a jet or an oblique fluid, the fluid direction changes, and the effect of different fluids is different. Fluid A moves near the jet boundary layer, and gradually merged with the jet, the jet's energy is also enhanced, fluid B is rising, continue to collide with other crossflows, jets, to the surface, has a greater impact on the liquid level. Fluid C, E, F in the form of eddy currents, and the $\mathrm{C}, \mathrm{E}$ is stronger, driven by the mainstream, to the surface, it has great influence on the stability of liquid surface [11-12], and F its energy is small, effect on the liquid level is less. Fluid D continue to move as a crossflow, and constantly colliding with other fluids, eventually it'll weaken, if the bath length tends to infinity, the comparison disappears with the decrease of its intensity during the fluid motion [13-14]. And for the collision of fluids in different directions in the bath, the superposition effect on the liquid level, will certainly increase the volatility of the liquid level. Therefore, in the coagulation bath, the interference caused by fluid collision cannot be ignored.

\subsection{Superimposed Coupling Effect of Fluid Wave}

In the solidification bath liquid surface, there must be the same direction or reverse of the fluid, that is, the overflow wave and reflux wave of the solidification bath fluid. in the opposite direction of the two fluids, the energy decreases with its length increasing with the source [15-17]. But the superposition coupling effect of wave peaks will increase the fluctuation of local and even the whole region, which will seriously affect the stability of liquid level. the superposition effect diagram is shown in figure 3 .

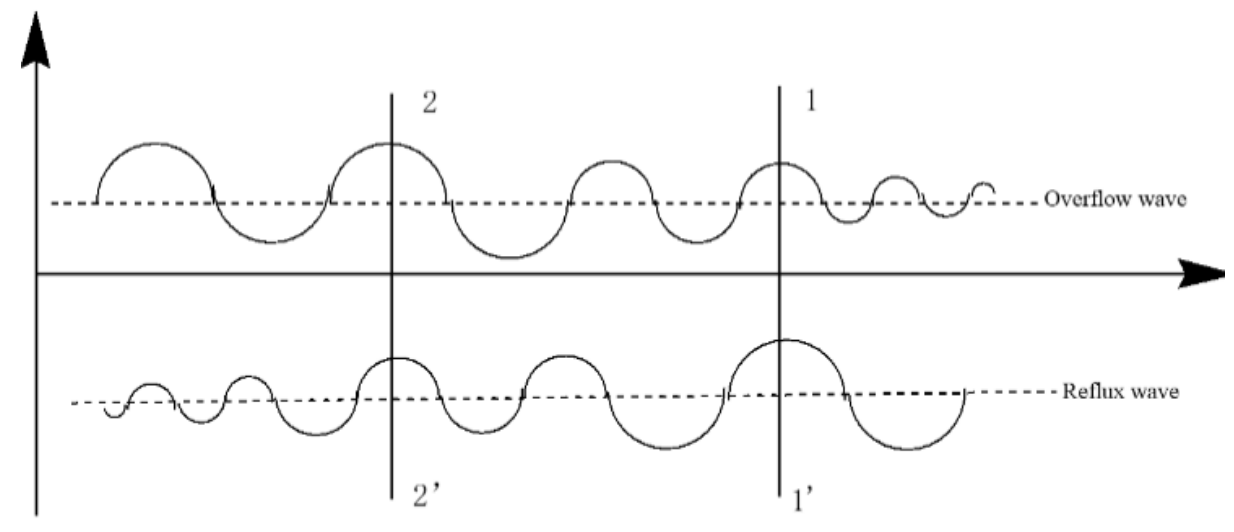

Figure 3. Superimposition of overflow and reflux waves.

It can be seen from Figure 3 that the intensity of overflow wave and reflux wave gradually decreases with its motion, but the superposition of the two waves during its motion. In Figure 3, the superposition coupling point of the two wave peaks, these two will strengthen the intensity, thus affecting the fluctuation of the liquid level. In the solidification bath, the superposition of different fluids is bound to aggravate the energy of the A, B, C, E fluid in Figure 2, which is very harmful.

\subsection{Interference of External Vibration Wave on Solidified Bath Liquid Surface}

In the production process, due to personnel operation, equipment operation and other inevitable vibration, the resulting vibration wave to the weak liquid level, is a fatal blow. The level of liquid increases with the intensity of vibration wave, which greatly increases the uncontrollability of dry spray wet solidification bath.

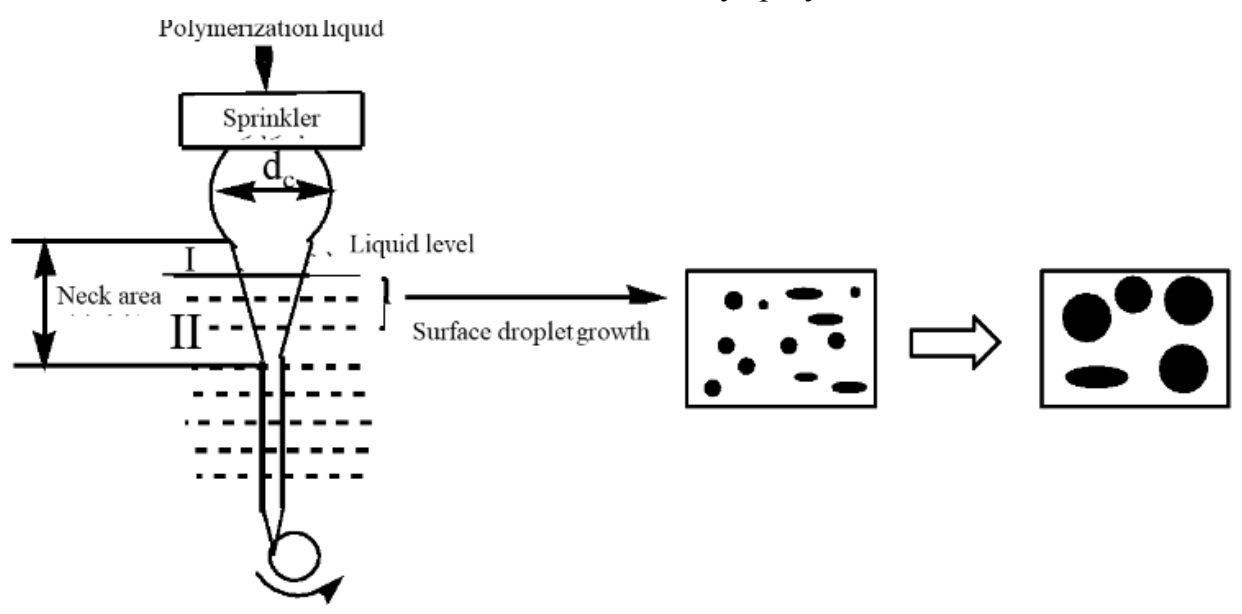

Figure 4. Dry jet wet spinning traction model. 


\subsection{Analysis of the Influence of the Fluctuation of Coagulation Bath Liquid Level on the Draft Consistency of Dry Jet Wet Spinning}

When the filament bundle enters the coagulation bath, the surface layer forms a dense film, and under the action of positive drawing, the neck will continue to form [18]. Due to the strong stretchability and moisture absorption of the film layer, the contact surface between the skin layer and the coagulation bath will increase during the necking process. At the same time, the dilute phase in the membrane rapidly diffuses outward, and under the action of the pressure difference, the dilute phase droplets have a tendency to increase. Therefore, at the initial stage of diffusion, a certain positive draft should be ensured. During the necking process, the dilute phase in the membrane cannot continue to increase under pressure, but rapidly solidifies through diffusion, thereby reducing the size of the pores.

It can be seen from the figure 4 that the pre-draft of dry and wet spinning is mainly concentrated in the neck area, and there is also a certain effective drafting area in the air layer. These two drafts play a decisive role in improving the spinning speed and the orientation of the raw yarn. Under the condition of a certain air layer height, the draft in the coagulation bath varies with the quenching depth of the coagulation bath, and the force of the filaments on the surface of the coagulation bath will change [19-21]. Therefore, the liquid level of the coagulation bath can be imagined as a steering roller. If the imaginary steering roller fluctuates, it will inevitably bring about inconsistencies in the length of the monofilament neck region, which will lead to poor monofilament consistency and decreased fiber running stability. Therefore, it is critical to study the coagulation bath level.

\section{Discussion on Fine Management Method of Liquid Level Fluctuation in Dry-spray Solid Bath}

Combined with the above research and analysis, the acquisition of high steady state solidification bath must depend on the fine management method. This paper discusses the fine management method of solidification bath liquid surface from personnel, equipment, technical method and environment [22-23].

\subsection{Fine Management - Technical Management}

1 Real-time monitoring. A high-precision infrared measuring instrument is used to quantitatively monitor the fluctuation amplitude of liquid level in real time. By relying on the PLC control system, the fluctuation amplitude of liquid level is displayed digitally in time. A flow rate tester is installed in the coagulation bath to monitor the flow rate and wavelength of key fluids in real time. With a single coagulation bath as a unit, a dynamic fluid movement model diagram is formed to keep abreast of the internal conditions of each bath.

2 Key technological breakthroughs. In the monitored big data, it is found that rising fluid is the main interference factor among the influencing factors of liquid level fluctuation, so reducing rising flow is the key. The fluid may also be clamped with bubbles and other substances during the rising process, relying on the key components and equipment to form a fluid damping control network centered on the solidification bath liquid surface, which can effectively control the motion trajectory and motion intensity of the fluid with interference to the liquid level in the tank, and realize the trend control of the fluid in the tank and the traceability of the trend data.

The stability of dry spray wet spinning nozzle is improved, the stability of spray nozzle is improved, and the fluctuation of solidification bath liquid surface caused by falling slurry and siphon is reduced. develop a long steady-state dry spray wet spinning nozzle stability control technology to reduce the nozzle replacement frequency and improve the continuous operation time of a single nozzle. At the same time, from the point of view of homogeneity and viscoelasticity of the original solution, the spinnability of the original solution is improved. Systematic formation of a dry spray wet spinning nozzle steady state control technology.

Process fine technical management system determination. A set of technical management methods suitable for the technical characteristics of enterprises is formed around the technical requirements, and the closed-loop control of technical management is formed, which can strengthen the comprehensive control of solidified bath liquid surface through the improvement of process management ability.

\subsection{Fine Management - Personnel Management}

The liquid level of dry-spray wet-spinning coagulation bath is an important part of spinning machine section. As the main body of production control and inspection, it is necessary to recognize the importance of this section, and to do "everything, starting from bit by bit" in the management of solidification bath liquid surface. Reasonable formulation of personnel inspection frequency, requirements, standards, after finding problems understand how to deal with, how to reduce fluctuations, how to quickly feedback, forming a set of standardized operation methods and rapid response mechanism. At the same time, in the process of management, the hidden trouble checking mechanism of the external cause of liquid level fluctuation is formed, and the external vibration wave interference factors of different production lines are not consistent, so we should find out and solve all the factors that may cause the change of wave intensity one by one, and form a continuous closed loop improvement.

\subsection{Fine Management - Equipment Management}

In the process of equipment design, we should consider the factors that may interfere with the liquid surface of solidification bath, such as the precision of driving equipment, 
the form and position of measuring equipment, the whole structure of spinning machine equipment, the seismic resistance of the whole equipment, etc. For such special working conditions, it is necessary to set up shock dampers in places with large vibration in the equipment, which can greatly reduce the influence of the outside on the fragile liquid level. It is necessary to do a good job in the daily cleaning, spot inspection, lubrication management of equipment related to the solidification bath liquid surface, so as to prevent the equipment from causing problems such as driving runout, running and leaking, and causing large liquid level fluctuation accidents. Equipment management should really achieve: prevention of micro-dudun gradually, fine management, achieve "equipment zero fault ".

A self-developed dry-jet wet spinning air layer height online real-time monitoring robot has the following functions:

1) Able to move in the dry jet and wet spinning section, detect the height of the air layer and transmit the information to the data terminal in real time.

2) Shock absorption damping is set on the robot body. The robot can absorb external shock waves in time during the patrol and monitoring process, avoiding the fluctuation of the solidification bath liquid level, and effectively ensuring the stability of the air layer.

3) The robot can move autonomously, realizing online real-time monitoring. The robot design includes moving components, lifting components, horizontal rotating components, damping, camera, detection unit and control unit.

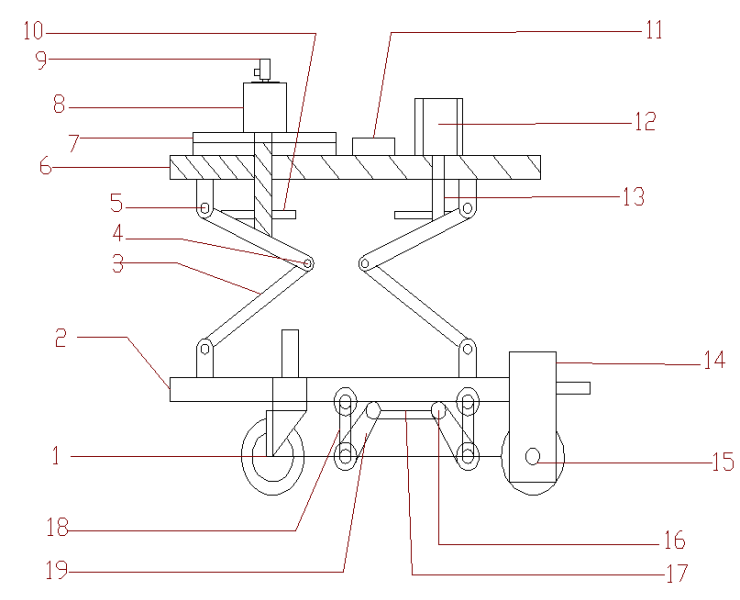

Figure 5. Monitoring robot.

In the figure 1. Driving wheel; 2. Lower support platform; 3. Support arm-1; 4 . Elevator steering gear; 5. Support arm-2; 6. Upper support platform; 7. Rotating platform; 8. Second rotation Steering gear; 9. Camera; 10. Big pulley; 11. Sensor; 12. First rotary steering gear; 13 . Small pulley; 14. Wheel carrier; 15. Drive wheel; 16. Slider; 17, Spring; 18. Shock absorption and damping; 19. Connecting rod.

\subsection{Fine Management - Environmental Control}

It was found in production that the influence of temperature and humidity on the liquid surface of solidification bath also has certain influence. temperature and humidity change, the effect of formed water droplets on the solidified bath liquid surface is fatal. Therefore, the environment control system of constant temperature and humidity is established to monitor the temperature and humidity in the workshop in real time, so as to reduce the interference to the steady state of solidified bath liquid surface caused by the change of external temperature and humidity.

\section{Conclusion}

The stability of solidified liquid level plays a decisive role in the stability and quality of dry spray wet spinning production, so it is very important to improve the stability of liquid level. Through the above technical analysis, combined with fine management methods, we can already achieve smooth control of liquid level.

The key to the quality control of carbon fiber lies in the control of detail, which can be said to be "detail determines success or failure ". With the gradual improvement of domestic carbon fiber technology maturity and the gradual expansion of production capacity, how to improve the control capacity of carbon fiber production line of 1000 tons or more in the future will become a new research topic. We believe that only around the quality-centered lean management, carbon fiber quality can be done better, the scale of the larger.

\section{Acknowledgements}

Transformation of S\&T achievements project of Jiangsu Province number BA2019043 thanks. Any opinions, findings, conclusions, or recommendations expressed in this material are those of the authors and do not necessarily reflect the views of the transformation of S\&T achievements project of Jiangsu Province.

\section{References}

[1] Yifeng, L.; Ximin, L. (2017). Meet the eve of strong development for carbon fiber industry. Hi-Tech Fiber \& Application, 42 (02): 1-9+16.

[2] Mingjin, W.; Hui, L.; Xiaogui, L.; Yongxiang, W.; Jianwen, X. (2013). The effect of coagulation floating field on the properties of PAN precursor. Carbon Techniques, 32 (03): 41-43.

[3] Yifeng, L. (2000). History of Development of PAN-based Carbon Fiber in China and Some Suggestions. Materials Reports, (04): 1-3.

[4] Xingguang, D. (2011). Study on the Effect of Coagulation Drawing on Polyacrylonitrile Nascent Fibers During Dry-jet Wet Spinning. Guangdong Chemical Industry, 38 (08): 48-50.

[5] Luo Yifeng; Ximin, L. (2015). Application developments of high performance and multifunctional light weight materials in defense equipments. Hi-Tech Fiber \& Application, 40 (06): $1-11+18$. 
[6] Liang, J.; Dong, L.; Keqing, Z.; Yi, S.; Jin, Z. (2013). Discussion on coagulation of dry-jet wet spinning of polyacrylonitrile precursor. Hi-Tech Fiber \& Application, 38 (06): 55-59.

[7] Youshou, T.; Huiqing, W.; Feng, L. (2011). The effect of coagulating bath on the performances of PAN precursor. Carbon Techniques, 30 (05): 14-16.

[8] Cohen C, Tanny G B. (1987). Diffusion-controlled formation of membranes by means of immersion precipitation: Part I. A model to describe mass transfer during immersion precipitation. Journal of Membrance Science, 34 (1): 45-65.

[9] Chen J C, Harrison I R. (2002) Modification of polyacrylonitrile (PAN) carbox fiber precursor via postspinning plasticization and stretching in dimethyl formamide (DMF). Carbox, 40 (1): 25-45.

[10] Ruijiao, D.; Marcus, K.; Jiongxin, Z.; Youwei, Z.; Chengxun, W.; Bing, P. (2008). Research progress on coagulation forming and phase separation of polyacrylonitrile precursor Synthesis Technology and Application, (02): 20-24.

[11] Guoqiang, J.; Wei, L. (2004). The vortex structure of a jet with a finite width and a narrow slot in a cross flow. Advances in Water Science, (05): 52-57+63.

[12] Wei, L.; Guoqiang, J.; Xiaoyuan, Z. (2003). Vortex structure of circular hole turbulent jet in cross flow. Advances in Water Science, (05): 576-582.

[13] Kim, S. Y.; Lee, S.; Park, S.; Jo, S. M.; Lee, H. S.; Joh, H. I. (2015). Continuous and rapid stabilization of polyacrylonitrile fiber bundles assisted by atmospheric pressure plasma for fabricating large-tow carbon fibers. Carbon, 94: 412-416.

[14] Li, J. H.; Chen, X. Q.; Li, X.; Cao, H. L.; Yu, H. Y.; Huang, Y. D. (2006). Synthesis, structure and properties of carbon nanotube/poly(p-phenylene benzobisoxazole) composite fibres. Polymer International, 55 (4): 456-465.

[15] Zeng, X. M.; Zhang, G.; Zhang, Y. W.; Zhao, J. X.; Pan, D.
(2006). Diffusion mechanism of as-spun polyacrylonitrile fiber in a dimethyl sulfoxide-water coagulation bath. Journal of Macromolecular Science Part a-Pure and Applied Chemistry, 43 (11): 1711-1720.

[16] Yu, D. G.; Teng, M. Y.; Chou, W. L.; Yang, M. C. (2003). Characterization and inhibitory effect of antibacterial PAN-based hollow fiber loaded with silver nitrate. Journal of Membrane Science, 225 (1-2): 115-123.

[17] Xu, L.; Zhang, L.; Chen, H. L. (2002). Study on $\mathrm{CO}_{2}$ removal in air by hydrogel membranes. Desalination, 148 (1-3): 309-313.

[18] Weisenberger, M. C.; Grulke, E. A.; Jacques, D.; Rantell, T.; Andrews, R. (2003). Enhanced mechanical properties of polyacrylonitrile/multiwall carbon nanotube composite fibers. Journal of Nanoscience and Nanotechnology, 3 (6): 535-539.

[19] Uchida, T.; Dang, T.; Min, B. G.; Zhang, X. F.; Kumar, S. (2005). Processing, structure, and properties of carbon nano fiber filled PBZT composite fiber. Composites Part B-Engineering, 36 (3): 183-187.

[20] Luo, K. Q.; Li, G.; Jin, J. H.; Yang, S. L.; Jiang, J. M. (2006). Surface analysis of PBO and modified SPBO fiber by contact angle measurements and XPS. Journal of Macromolecular Science Part B-Physics, 45 (4): 631-637.

[21] Kumar, S.; Dang, T. D.; Arnold, F. E.; Bhattacharyya, A. R.; Min, B. G.; Zhang, X. F.; Vaia, R. A.; Park, C.; Adams, W. W.; Hauge, R. H.; Smalley, R. E.; Ramesh, S.; Willis, P. A. (2002). Synthesis, structure, and properties of $\mathrm{PBO} / \mathrm{SWNT}$ composites. Macromolecules, 35 (24): 9039-9043.

[22] Chou, W. L.; Yu, D. G.; Yang, M. C. (2005). The preparation and characterization of silver-loading cellulose acetate hollow fiber membrane for water treatment. Polymers for Advanced Technologies, 16 (8): 600-607.

[23] Bajaj, P.; Sreekumar, T. V.; Sen, K. (2002). Structure development during dry-jet-wet spinning of acrylonitrile/vinyl acids and acrylonitrile/methyl acrylate copolymers. Journal of Applied Polymer Science, 86 (3): 773-787. 